\title{
SUBORDINATION CHAINS AND QUASICONFORMAL EXTENSION OF HOLOMORPHIC MAPS IN $C^{n}$
}

\author{
J. A. PFAltzgrafF
}

\section{Introduction}

Recently [1] J. Becker showed that a function $f(z)$ analytic in $|z|<1$ extends to a quasiconformal homeomorphism of $C$ onto $C$ if it satisfies

$$
\left(1-|z|^{2}\right)\left|z f^{\prime \prime}(z) / f^{\prime}(z)\right| \leq c<1,|z|<1 .
$$

In this paper we give the $n$-dimensional $(n>1)$ generalization of Becker's extension theorem. We shall use results and notation established in [7].

Let $\mathcal{X}\left(B^{n}\right)$ denote the set of functions

$$
f(z)=\left(f_{1}(z), \ldots, f_{n}(z)\right), z=\left(z_{1}, \ldots, z_{n}\right),
$$

that are holomorphic in $B^{n}=\left\{z \in C^{n}:\|z\|<1\right\}$ with values in $C^{n}$. We consider $C^{n}$ with the usual inner product, $\langle$,$\rangle , and Euclidean norm \|\|$. The second derivative of a function $f \in \mathcal{H}\left(B^{n}\right)$ is a symmetric bilinear operator $D^{2} f(z)(\cdot, \cdot)$ on $C^{n} \times C^{n}$, and $D^{2} f(z)(z, \cdot)$ is the linear operator obtained by restricting $D^{2} f(z)$ to $z \times C^{n}$.

We shall prove (Theorem 3.1) that if $f \in \mathcal{X}\left(B^{n}\right)$ is quasiregular in $B^{n}$ and if there is a constant $c<1$ such that

$$
\left(1-\|z\|^{2}\right)\left\|(D f(z))^{-1} D^{2} f(z)(z, \cdot)\right\| \leq c, \quad z \in B^{n},
$$

then $f$ extends to a quasiconformal homeomorphism of $R^{2 n}$ onto $R^{2 n}$. As in [1] the extension is given explicitly in terms of an appropriate subordination chain ((3.3) below) already considered in Theorem 2.4 of [7]. In (1.2) \|\| is the usual operator norm

$$
\|A\|=\sup \{\|A z\|:\|z\| \leq 1\}
$$

for $A \in \mathscr{L}\left(C^{n}\right)$, the continuous linear operators from $C^{n}$ to $C^{n}$.

The matrix representation for $D^{2} f(z)\left(z,,^{\cdot}\right)$ is 


$$
D^{2} f(z)(z, \cdot)=\left(\sum_{m=1}^{n} \frac{\partial^{2} f_{k}(z)}{\partial z_{j} \partial z_{m}} z_{m}\right), \quad 1 \leq j, k \leq n .
$$

For $n=1$, this reduces to $z f^{\prime \prime}(z)$ and (1.2) reduces to Becker's condition (1.1).

In Section 2 we prove some preliminary results on Lipschitz continuous extension to the closed ball, $\bar{B}^{n}$. These results are independent of the theory of subordination chains.

\section{Lipschitz continuous extension}

Our first result (Lemma 2.1) is an $n$-dimensional version of classical theorems of Hardy and Littlewood [3, pp. 361-363]. We then apply this result in Theorem 2.1 to the problem of continuous extension to $\bar{B}^{n}$ of a locally biholomorphic quasiconformal mapping on $B^{n}$.

We say that a holomorphic mapping $f(z)=\left(f_{1}(z), \ldots, f_{n}(z)\right)$ from a domain $G \subset C^{n}$. into $C^{n}$ is quasiregular in $G$ if

$$
\|D f(z)\|^{n} \leq K|\operatorname{det} D f(z)|
$$

for some $K<\infty$ and all $z \in G$. It is known that a sufficiently smooth quasiregular mapping is a local homeomorphism. Hence a quasiregular holomorphic mapping has nonsingular $D f(z)$ and is automatically locally biholomorphic. Note that $n$ in (2.1) is the complex dimension, but the holomorphy of $f$ makes it easy to interpret (2.1) as a condition on mappings in $R^{2 n}$ in the notation of [9]. We shall return to this point in Section 3 below.

L e m m a 2.1. Suppose that $c \in[0,1)$ and that $g(z)$ is a complex valued holomorphic function for $z \in B^{n}$, such that

$$
\left|\frac{\partial g(z)}{\partial z_{j}}\right| \leq M_{j} /(1-\|z\|)^{c}, \quad j=1, \ldots, n, \quad z \in B^{n} .
$$

Then $g(z)$ has a continuous extension to $\bar{B}^{n}$ and

$$
|g(z)-g(w)| \leq A\|z-w\|^{1-c}, \quad z, w \in \bar{B}^{n} .
$$

Proof. Note that $D g(z)=\left(\partial g(z) / \partial z_{1}, \ldots, \partial g(z) / \partial z_{n}\right)$ and the conditions (2.2) imply

$$
\|D g(z)\| \leq M /(1-\|z\|)^{c}, \quad z \in B^{n}, \quad M=\left\{\sum_{j=1}^{n} M_{j}^{2}\right\}^{\frac{1}{2}} .
$$

Fix a point $a \in C^{n}$ on the sphere $\|a\|=1$ and consider the slice function $G_{a}(\zeta)=g(\zeta a), \quad \zeta \in B^{1}$. Then $G_{a}(\zeta)$ is holomorphic in $|\zeta|<1$, and satisfies 


$$
\begin{gathered}
\left|G_{a}^{\prime}(\zeta)\right|=|\langle D g(\zeta a), a\rangle| \leq\|D g(\zeta a)\| \leq \\
\leq M /(1-\|\zeta a\|)^{c}=M /(1-|\zeta|)^{c}, \quad|\zeta|<1
\end{gathered}
$$

(recall that $\langle z, w\rangle=\sum_{j=1}^{n} z_{j} \bar{w}_{j}, z, w \in C^{n}$ ). Hence $G_{a}(\zeta)$ is continuous in the closed unit disk (Satz 3 [3, p. 361]) and satisfies a complex Lipschitz condition

$$
\left|G_{a}(\zeta)-G_{a}\left(\zeta^{\prime}\right)\right| \leq k\left|\zeta-\zeta^{\prime}\right|^{1-c}, \quad \zeta, \zeta^{\prime} \in \bar{B}^{1}
$$

in the closed disk [3, pp. 361-364]. An examination of the proofs of Satz 3 and Satz 4 in [3, pp. 361-364] shows that the constant $k$ in (2.4) depends only on $M$ and $c$, and is independent of the choice of $a \in C^{n},\|a\|=1$. We extend $g$ to the boundary of $B^{n}$ by defining $g(z)=G_{z}(1)$ for each $z$ on $\|z\|=1$.

We let $z \in \bar{B}^{n}, \quad 0 \leq h \leq 1$ and apply (2.4) with $a=z /\|z\|, \quad \zeta=\|z\|$ to obtain

$$
|g(z)-g(h z)| \leq k|\|z\|-h\|z\||^{1-c} \leq k(1-h)^{1-c} .
$$

For any pair of points $w, w^{\prime} \in B^{n}$ we also have

$$
\begin{gathered}
\left|g(w)-g\left(w^{\prime}\right)\right| \leq\left\|w^{\prime}-w\right\| \int_{0}^{1}\left\|D g\left(w+t\left(w^{\prime}-w\right)\right)\right\| d t \\
\leq M\left\|w^{\prime}-w\right\| /\left(1-\max \left(\|w\|,\left\|w^{\prime}\right\|\right)\right)^{c}
\end{gathered}
$$

To complete the proof we first show that there is a constant $L$ independent of $r, 0<r \leq 1$, such that

$$
|g(z)-g(w)| \leq L\|z-w\|^{1-c}
$$

when $\|z\|=\|w\|=r$. Let $\|z\|=\|w\|=r \leq 1,0 \leq h<1$, and apply (2.5) and (2.6) to obtain

$$
|g(z)-g(w)| \leq|g(z)-g(h z)|+|g(w)-g(h w)|+|g(h z)-g(h w)|
$$

$$
\leq 2 k(1-h)^{1-c}+M\|z-w\| /(1-h r)^{c} \leq 2 k(1-h)^{1-c}+M\|z-w\| /(1-h)^{c} .
$$

To prove (2.7) for a fixed $r \leq 1$ it is sufficient to do this for $\|z-w\|<1$. Setting $h=1-\|z-w\|$ in (2.8) we obtain

$$
|g(z)-g(w)| \leq(2 k+M)\|z-w\|^{1-c}
$$

when $\|z\|=\|w\|=r \leq 1$ and $\|z-w\|<1$. An obvious compactness argument shows that (2.7) holds for all $r \leq 1$ with a constant $L$ that is independent of $r$. Finally we let $z, w \in \bar{B}^{n}$ and suppose that $\|w\| \leq\|z\| \leq 1$. Then 


$$
\begin{gathered}
|g(z)-g(w)| \leq|g(z)-g(\|z\| w /\|w\|)|+|g(\|z\| w /\|w\|)-g(w)| \\
\leq L\|z-\| z\|w /\| w\|\|^{1-c}+k|\|z\|-\|w\||^{1-c} \leq\left(L 2^{1-c}+k\right)\|z-w\|^{1-c}
\end{gathered}
$$

by (2.5), (2.7) and the triangle inequality. The proof of Lemma 2.1 is complete.

Prior to the writing of this paper J. Mitchell proved a general version of Lemma 2.1 for bounded symmetric domains in $C^{n}$ [4]. I am indebted to J. Mitchell for discussing with me her results in [4].

L e m m a 2.2. Let $f(z)=\left(f_{1}(z), \ldots, f_{n}(z)\right) \in X\left(B^{n}\right)$ and suppose that

$$
\|D f(z)\|=O\left(1 /(1-\|z\|)^{c}\right),\|z\|<1, \quad 0 \leq c<1 .
$$

Then $f$ has a continuous extension to $\bar{B}^{n}$ and

$$
\|f(z)-f(w)\| \leq A\|z-w\|^{1-c}, z, w \in \bar{B}^{n} .
$$

Proof. It suffices to note that (2.9) yields conditions of the form (2.2) on each of the components $f_{j}(z), j=1, \ldots, n$ and then apply Lemma 2.1 to each of these functions.

Theorem 2.1. Let $f(z) \in \mathcal{C}\left(B^{n}\right)$ be quasiregular in $B^{n}$. If there is a constant $c<1$ such that

$$
\left\|(D f(z))^{-1} D^{2} f(z)(z, \cdot)\right\| \leq \frac{2 c}{1-\|z\|^{2}}
$$

for all $z \in B^{n}$, then

$$
\|D f(z)\|=O\left(1 /(1-\|z\|)^{c}\right), \quad z \in B^{n},
$$

and $f$ has a Lipschitz continuous extension to $\bar{B}^{n}$ that satisfies

$$
\|f(z)-f(w)\| \leq M\|z-w\|^{1-c}, \quad z, w \in \bar{B}^{n} .
$$

Proof. By Lemma 2.2 it is sufficient to prove (2.11). We fix $w \in \bar{B}^{n}$ and let $A(\zeta), \zeta \in B^{1}$, denote the holomorphic matrix-valued function $A(\zeta)=D f(w \zeta)$. An easy computation shows that

$$
\zeta A^{\prime}(\zeta)=\left(\sum_{m=1}^{n} \frac{\partial^{2} f_{k}(w \zeta)}{\partial z_{j} \partial z_{m}} w_{m} \zeta\right)=D^{2} f(w \zeta)(w \zeta, \cdot)
$$

The complex valued function $g(\zeta)=\operatorname{det} A(\zeta)$ is a nonvanishing holomorphic function of $\zeta$ in the open unit disk. Employing an argument of M. A. Goldberg [2] we find that

$$
g^{\prime}(\zeta)=g(\zeta) \operatorname{tr}\left[A^{-1}(\zeta) A^{\prime}(\zeta)\right]
$$

where $\operatorname{tr}$ denotes the trace, and therefore

$$
\zeta g^{\prime}(\zeta) / g(\zeta)=\operatorname{tr}\left[(D f(w \zeta))^{-1} D^{2} f(w \zeta)(w \zeta, \cdot)\right]
$$


If $E=\left(E_{j k}\right) \in \mathscr{L}\left(C^{n}\right)$ then $\|E\|^{2}=\lambda_{n}$ where $\lambda_{1} \leq \lambda_{2} \leq \ldots \leq \lambda_{n}$ are the eigenvalues of $E^{*} E\left(E^{*}\right.$ denotes the conjugate transpose). It is elementary to show that

$$
\operatorname{tr}\left(E^{*} E\right)=\sum_{j, k=1}^{n}\left|E_{j k}\right|^{2}=\lambda_{1}+\ldots+\lambda_{n} \leq n \lambda_{n}=n\|E\|^{2},
$$

and hence that

$$
|\operatorname{tr} E|=\left|\sum_{j=1}^{n} E_{j j}\right| \leq\left\{n \sum_{j=1}^{n}\left|E_{j j}\right|^{2}\right\}^{1 / 2} \leq n\|E\| .
$$

We apply $(2.10)$ and $(2.14)$ to $(2.13)$ to obtain

$$
\left|\zeta \frac{d}{d \zeta} \log g(\zeta)\right| \leq 2 n c /\left(1-\|w \zeta\|^{2}\right) \leq 2 n c /\left(1-|\zeta|^{2}\right)
$$

for $|\zeta|<1$, and therefore

$$
|\operatorname{det} D f(w \zeta)|=|g(\zeta)|=O\left(1 /(1-|\zeta|)^{n c}\right), \quad|\zeta|<1 .
$$

Next, we let $z \in B^{n}$ and apply (2.15) with $w=z /\|z\|, \zeta=\|z\|$, to conclude that

$$
|\operatorname{det} D f(z)|=O\left(1 /(1-\|z\|)^{n c}\right), \quad z \in B^{1} .
$$

Finally, (2.16) and the quasiregularity condition (2.1) imply that (2.11) holds, and the proof of the theorem is complete.

Example. Let $z=\left(z_{1}, z_{2}\right)$ and define

$$
f(z)=\left(z_{1}, z_{2}+h\left(z_{1}\right)\right), \quad z \in B^{2},
$$

where $h(0)=0=h^{\prime}(0)$ and $h$ is analytic in $\left|z_{1}\right|<1$, but otherwise arbitrary. Then $f$ is univalent throughout $B^{2}$ (not just locally), and easy calculation shows that

$$
L(f ; z)=(D f(z))^{-1} D^{2} f(z)(z, \cdot)=\left(\begin{array}{cc}
0 & 0 \\
z_{1} h^{\prime \prime}\left(z_{1}\right) & 0
\end{array}\right) .
$$

Thus trace $L(f ; z)=0$, but $f$ may not extend to $\bar{B}^{2}$ for arbitrary $h$. However (2.10) obviously restricts $h^{\prime \prime}\left(z_{1}\right)$ enough to render $h\left(z_{1}\right)$ extendable and Lipschitz continuous. We also note that

$$
D f(z)=\left(\begin{array}{cc}
1 & 0 \\
h^{\prime}\left(z_{1}\right) & 1
\end{array}\right)
$$

$\operatorname{det} D f(z)=1$, and, by calculating the eigenvalues of $(D f)^{*} D f$,

$$
\|D f(z)\|^{2}=2^{-1}\left\{\left|h^{\prime}\left(z_{1}\right)\right|^{2}+2+\left(\left|h^{\prime}\left(z_{1}\right)\right|^{4}+4\left|h^{\prime}\left(z_{1}\right)\right|^{2}\right)^{1 / 2}\right\} .
$$

Hence (2.17) is quasiregular only if $h\left(z_{1}\right)$ has bounded derivative in $\left|z_{1}\right|<1$. 
Acknowledgement. We wish to thank Carlos Berenstein for reading the original manuscript and making a helpful comment on Theorem 2.1.

\section{Quasiconformal extension}

In [7] (Theorem 2.4) we showed that if $f \in \mathcal{C}\left(B^{n}\right)$ is locally biholomorphic and (1.2) holds in $B^{n}$ for $c \leq 1$ then $f$ is univalent in $B^{n}$. We now wish to show that if condition (1.2) with $c<1$ holds then $f$ is not only univalent in $B^{n}$, but it extends to a quasiconformal homeomorphism of $R^{2 n}$ onto $R^{2 n}$. The extended mapping $f=\left(f_{1}, \ldots, f_{n}\right)$ may not be holomorphic outside $B^{n}$, and hence will be considered as a mapping

$$
f:\left(x_{1}, y_{1}, \ldots, x_{n}, y_{n}\right) \mapsto\left(u_{1}, v_{1}, \ldots, u_{n}, v_{n}\right)
$$

of $R^{2 n}$ to $R^{2 n}$ where $z_{k}=x_{k}+i y_{k} \quad$ and $\quad u_{k}=\operatorname{Re} f_{k}, \quad v_{k}=\operatorname{Im} f_{k}$, $k=1, \ldots, n$. We shall show that the extension is ACL, (real) differentiable a.e., and has uniformly bounded dilatation a.e., and is therefore quasiconformal according to [9, p. 115]. The (real) derivative $(2 n \times 2 n$ Jacobian matrix) of the mapping $f$ in (3.1) will be denoted by $D(u, v ; x, y)$ and $J(u, v ; x, y)$ will denote the Jacobian determinant of $f$. At points where $f$ is differentiable but not complex holomorphic we shall consider the (outer) dilatation,

$$
\|D(u, v ; x, y)\|^{2 n} / J(u, v ; x, y),
$$

of $f$, where \|\| is the standard operator norm for $2 n \times 2 n$ real matrices. Concerning notation, if $z=\left(z_{1}, \ldots, z_{n}\right) \in C^{n}$ and $z_{j}=x_{j}+i y_{j}$, $1 \leq j \leq n$, then $(x, y)$ will denote the representation $(x, y)=$ $\left(x_{1}, y_{1}, \ldots, x_{n}, y_{n}\right)$ of $z$ as a row vector in $R^{2 n}$, and $\left(\begin{array}{l}x \\ y\end{array}\right)$ will denote the (real) column vector that is the transpose of $(x, y)$. It will be necessary to use the real notation rather than the more concise complex notation when the mappings in question are not complex holomorphic.

Before proceeding to our main theorem we must recall some of the basic definitions and results from [7]. The notation $f(z)=a z+\ldots, a \in C$, for $f \in \mathcal{X}\left(B^{n}\right)$ indicates that $D f(0)=a I$ where $I=\left(I_{j k}\right)$ is the identity in $\mathscr{L}\left(C^{n}\right)$. A mapping $v(z) \in \mathcal{C}\left(B^{n}\right)$ is called a Schwarz function if $\|v(z)\| \leq\|z\|$ for all $z \in B^{n}$. A subordination chain ([7], [8]) is a family of functions $\left\{f(z, t): z \in B^{n}, t \geq 0\right\}$ from $B^{n} \times[0, \infty)$ into $C^{n}$ such that for each $t \geq 0, f(z, t)=e^{t} z+\ldots$ is in $\mathcal{C}\left(B^{n}\right)$ and there exist Schwarz functions $v(z, s, t)$ such that

$$
f(z, s)=f(v(z, s, t), t), \quad 0 \leq s \leq t, \quad z \in B^{n}
$$


for all $0 \leq s \leq t<\infty$.

In [7] (Theorem 2.4) we defined

$$
f(z, t)=f\left(z e^{-t}\right)+\left(e^{t}-e^{-t}\right) D f\left(z e^{-t}\right)(z), \quad t \geq 0,
$$

for locally biholomorphic $f(z)=z+\ldots$ in $\mathcal{X}\left(B^{n}\right)$ such that (1.2) holds for $c \leq 1$. From (3.3) we calculated

$$
e^{-t} D f(z, t)=D f\left(z e^{-t}\right)[I-E(z, t)]
$$

where for each $(z, t) \in B^{n} \times[0, \infty), E(z, t)$ is the linear operator

$$
E(z, t)=-\left(1-e^{-2 t}\right)\left(D f\left(z e^{-t}\right)\right)^{-1} D^{2} f\left(z e^{-t}\right)\left(z e^{-t}, \cdot\right) .
$$

From (1.2) we have $\|E(z, t)\| \leq c$ and then $\|E(z, t)\| \leq c\|z\|$ by the general Schwarz lemma $\left(E(z, t)\right.$ is a bounded holomorphic mapping into $\left.\mathscr{L}\left(C^{n}\right)\right)$.

The functions (3.3) satisfy the generalized Loewner differential equation $([7],[6])$

$$
\frac{\partial f}{\partial t}(z, t)=f(z, t) h(z, t), \quad t \geq 0, \quad z \in B^{n}
$$

where

$$
h(z, t)=(I-E(z, t))^{-1}(I+E(z, t))(z),
$$

and as a consequence ([7], Theorem 2.2), (3.3) is a subordination chain with $v(z, s, t)$ in (3.2) being univalent Schwarz functions generated as the unique solutions to the initial value problems

$$
\frac{\partial v(z, s, t)}{\partial t}=-h(v(z, s, t), t), \quad t \geq s, \quad v(z, s, s)=z
$$

We will also need the following bounds established in [7]:

$$
\begin{gathered}
\|h(z, t)\| \leq\|z\|(1+c\|z\|) /(1-c\|z\|), \quad z \in B^{n}, \\
\|z\|^{2}(1-c\|z\|) /(1+c\|z\|) \leq \operatorname{Re}\langle h(z, t), z\rangle, \quad z \in B^{n} .
\end{gathered}
$$

The or e $\mathrm{m}$ 3.1. Let $f(z)=z+\ldots$ be holomorphic and quasiregular in $B^{n}$. If there is a constant $c<1$ such that

$$
\left(1-\|z\|^{2}\right)\left\|(D f(z))^{-1} D^{2} f(z)(z, \cdot)\right\| \leq c
$$

for all $z \in B^{n}$ then $f$ can be extended to a quasiconformal homeomorphism of $R^{2 n}$ onto $R^{2 n}$. The extension is given by

$$
F(z)=\left\{\begin{array}{l}
f(z, 0), \quad\|z\| \leq 1 \\
f(z /\|z\|, \quad \log \|z\|), \quad\|z\|>1
\end{array}\right.
$$

where $\{f(z, t)\}$ is the subordination chain 


$$
f(z, t)=f\left(z e^{-t}\right)+\left(e^{t}-e^{-t}\right) D f\left(z e^{-t}\right)(z), \quad t \geq 0 .
$$

Proof. We imbed $f(z)$ as the initial element in the chain (3.3). The condition (3.12) and our univalence results in [7] (Theorem 2.3) yield that each $f_{t}(z)=f(z, t) \quad(t \geq 0)$ is univalent in $B^{n}$. Furthermore, for $t>0$, $f(z, t)$ is obviously holomorphic in $\bar{B}^{n}$, and $f(z, 0)=f(z)$ is continuous in $\bar{B}^{n}$ by (3.11) and Theorem 2.1 on Lipschitz extensions. We shall show that $f(z, t) \quad(t \geq 0)$ is univalent in $\bar{B}^{n}$, and that $f_{s}\left(\bar{B}^{n}\right) \subset f_{t}\left(B^{n}\right)$ when $0 \leq s<t$. Then it will be obvious that the extension $F(z)$ in (3.13) is univalent in $C^{n}\left(R^{2 n}\right)$.

In [7] we showed that the Schwarz functions $v=v(z, s, t)$ in (3.8) satisfy

$$
\|v\| \partial\|v\| / \partial t=-\operatorname{Re}\langle h(v, t), v\rangle
$$

and hence

$$
\|v(z, s, t)\|^{-1} \partial\|v(z, s, t)\| / \partial t \leq-\frac{(1-c)}{(1+c)}, \quad s<t
$$

by (3.10). An elementary integration and the initial condition $v(z, s, s)=z$ yield the inequality

$$
\|v(z, s, t)\| \leq\|z\| \exp \left\{-(1-c)(1+c)^{-1}(t-s)\right\}, \quad 0 \leq s<t .
$$

Hence $\overline{v\left(B^{n}, s, t\right)} \subset B^{n} \quad(0 \leq s<t)$, and this combined with the continuity of $f_{s}(z)$ in $\bar{B}^{n}, f_{t}(z)$ in $B^{n}$, and the relation $f_{s}(z)=f_{t}(v(z, s, t))$ shows that $f_{s}\left(\bar{B}_{,}^{n}\right) \subset f_{t}\left(B^{n}\right), 0 \leq s<t$ (cf. [1, p. 32]). The continuity of $f_{s}(z)$ in $\bar{B}^{n}$ enables us to define the continuous extension $v(z, s, t)=$ $f_{t}^{-1}\left(f_{s}(z)\right) \quad\left(z \in \bar{B}^{n}, 0 \leq s<t\right)$ of $v(z, s, t)$ to $\bar{B}^{n}$. To show that $f_{s}$ is univalent in $\bar{B}^{n}$ we first note, for $z \in B^{n}$ and $0 \leq s<t$, that

$$
\begin{gathered}
\|z-v(z, s, t)\|=\left\|\int_{s}^{t} h(v(z, s, \tau), \tau) d \tau\right\| \\
\leq \int_{s}^{t}\|v\|(1+c\|v\|) /(1-c\|v\|) d \tau \leq(1+c)(1-c)^{-1}(t-s)
\end{gathered}
$$

(by (3.8) and (3.9)). It is easy to show that (3.15) is valid in $\bar{B}^{n}$ for the extended $v(z, s, t)$. The univalence of $f_{s}(z)(s \geq 0)$ in $\bar{B}^{n}$ now follows from (3.15) by a brief argument given in [1, p. 32].

Having established the univalence of $F$ in $C^{n}$, we now turn to the question of continuity in $C^{n}$. The continuity of $F$ in $C^{n}$ will follow from the Lipschitz conditions

$$
\begin{gathered}
e^{-t}\|f(z, t)-f(w, t)\| \leq M\|z-w\|^{1-c}, \quad z, w \in \bar{B}^{n}, \quad t \geq 0 \\
\|f(z, t)-f(z, s)\| \leq L e^{t}(t-s)^{1-c}, \quad z \in \bar{B}^{n}, \quad 0 \leq s<t
\end{gathered}
$$


( $M$ and $L$ are constants independent of $s$ and $t$.) To prove (3.16) we begin with (3.4). The quasiconformality of $f(z),(3.11)$, and Theorem 2.1 $((2.11))$ then yield that

$$
\left\|e^{-t} D f(z, t)\right\| \leq\left\|D f\left(z e^{-t}\right)\right\|(1+c) \leq M^{\prime} /(1-\|z\|)^{c},
$$

for all $z \in B^{n}$ and $t \geq 0$, where $M^{\prime}$ is a constant independent of $t$. Hence the functions $e^{-t} f(z, t) \quad(t \geq 0)$ satisfy (2.9), and (3.16) follows from Lemma 2.2. To prove (3.17) we first observe that $\|f(z, t)-f(z, s)\|=$ $\|f(z, t)-f(v(z, s, t), t)\| \leq M e^{t}\|z-v(z, s, t)\|^{1-c}$, for $z \in \bar{B}^{n}, 0 \leq s<t$, and then apply (3.15). The continuity in $C^{n}$ of the extension (3.13) follows since (3.16) and (3.17) show that $f(z, t)$ is continuous in $\bar{B}^{n} \times[0, \infty)$.

In [7] we showed that the Schwarz functions $v=v(z, s, t)$ in (3.8) satisfy the inequalities:

$$
e^{t}\|v(z, s, t)\| /(1-c\|v(z, s, t)\|)^{2} \leq e^{s}\|z\| /(1-c\|z\|)^{2},
$$

and

$$
e^{s}\|z\| /(1+c\|z\|)^{2} \leq e^{t}\|v(z, s, t)\| /(1+c\|v(z, s, t)\|)^{2},
$$

and that $e^{t} v(z, s, t)$ converges to $f(z, s)$ as $t \rightarrow \infty$ with $s$ fixed. Thus we let $t \rightarrow \infty$ to obtain

$$
e^{s}\|z\| /(1+c\|z\|)^{2} \leq\|f(z, s)\| \leq e^{s}\|z\| /(1-c\|z\|)^{2} .
$$

The left-hand inequality (3.18) shows that $F(z) \rightarrow \infty$ as $z \rightarrow \infty$, and hence that $F(z)$ in (3.13) is a homeomorphism of $R^{2 n} U\{\infty\}$ onto itself. It remains to show that $F$ is quasiconformal in $R^{2 n}$. We shall do this with an approximation argument similar to Becker's [1, pp. 33-34].

We let $r>1$ and define the functions $f_{r}(z, t)=r f(z / r, t) \quad(t \geq 0)$, $h_{r}(z, t)=r h(z / r, t)$ and

$$
G_{r}(z)=\left\{\begin{array}{l}
f_{r}(z, 0), \quad\|z\| \leq 1 \\
f_{r}(z /\|z\|, \log \|z\|), \quad\|z\| \geq 1 .
\end{array}\right.
$$

Clearly, $f_{r}(z, t)$ satisfies the differential equation

$$
\partial f_{r}(z, t) / \partial t=D f_{r}(z, t) h_{r}(z, t), t \geq 0,
$$

for all $\|z\|<r$, and hence $\|z\| \leq 1$. Furthermore one can use (3.16) and the right-hand side of (3.18) to show that $f_{r}(z, t) \rightarrow f(z, t)$ uniformly in $\|z\| \leq 1,0 \leq t \leq T$, as $r$ decreases to 1 . Hence $G_{r}(z)$ converges to $F(z)$ uniformly on compacta in $R^{2 n}$ as $r$ decreases to 1 . We shall show that $G_{r}(z)$ (as a mapping from $R^{2 n}$ to $R^{2 n}$ ) is ACL, differentiable a.e., and has outer dilation bounded a.e. by a bound independent of $r$. Then it will follow (see 21.3 and 37.4 in [9]) that $F$ is quasiconformal.

Clearly $e^{-t} f_{r}(z, t)$ satisfies a Lipschitz condition 


$$
e^{-t}\left\|f_{r}(z, t)-f_{r}(w, t)\right\| \leq M_{r}\|z-w\|, \quad z, w \in \bar{B}^{n}, t \geq 0,
$$

where $M_{r}>0$ depends only upon $r>1$, since $\left\|e^{-t} D f_{r}(z, t)\right\|=$ $\left\|e^{-t} D f(z / r, t)\right\| \leq\left\|D f\left(e^{-t} z / r\right)\right\|(1+c)$ and this last quantity (for fixed $r>1$ ) is uniformly bounded for all $z \in \bar{B}^{n}$ and $t \geq 0$. Moreover, similar to (3.17), we have that

$$
\left\|f_{r}(z, t)-f_{r}(z, s)\right\| \leq e^{t} L_{r}(t-s), \quad z \in \bar{B}^{n}, \quad 0 \leq s \leq t,
$$

where we have used (3.21) and (3.15). With (3.21) and (3.22) it is easy to show that $G_{r}(z)$ satisfies a local Lipschitz condition (with exponent 1) in $C^{n}$. Hence $G_{r}(z)$ is ACL in $R^{2 n}$, and by the Rademacher-Stepanov theorem [9, p. 97] it is (real) differentiable a.e. in $R^{2 n}$.

Throughout the remainder of this proof we shall simplify notation by dropping the subscript $r$ and letting $G(z)=G_{r}(z)$ for fixed $r>1$. We let $z=(x, y), \quad\|z\| \geq 1$, be a point where the mapping $G=(U, V)$,

$$
G:\left(x_{1}, y_{1}, \ldots, x_{n}, y_{n}\right) \mapsto\left(U_{1}, V_{1}, \ldots, U_{n}, V_{n}\right),
$$

$$
U_{k}=U_{k}\left(x_{1}, y_{1}, \ldots, x_{n}, y_{n}\right)=\operatorname{Re} G_{k}(x, y), \quad V_{k}=\operatorname{Im} G_{k}(x, y),
$$

$k=1, \ldots, n$, is differentiable. We shall complete the proof by showing that

$$
\|D(U, V ; x, y)\|^{2 n} \leq K^{2}\left(\frac{1+c}{1-c}\right)^{4 n-1}|J(U, V ; x, y)| .
$$

To compute the (real) derivative of (3.23) one uses the chain rule on the composed mapping (see (3.19))

$\left(x_{1}, y_{1}, \ldots, x_{n}, y_{n}\right) \mapsto\left(\xi_{1}, \eta_{1}, \ldots, \xi_{n}, \eta_{n}, \log \|z\|\right) \mapsto r\left(u_{1}, v_{1}, \ldots, u_{n}, v_{n}\right)$, where $\zeta_{k}=\xi_{k}+i \eta_{k}=z_{k} /(r\|z\|), \quad \zeta=z /(r\|z\|), \quad u_{k}$ and $v_{k}$ are the real and imaginary parts of $f_{k}(\zeta, t)$, and $U_{k}(x, y)=r u_{k}(\xi, \eta, t), \quad V_{k}(x, y)=$ $r v_{k}(\xi, \eta, t)$ with $t=\log \|z\|$. Elementary computations yield that

$$
\begin{gathered}
D(U, V ; x, y)=r\left\{D(u, v ; \xi, \eta) D(\xi, \eta ; x, y)+\frac{\partial}{\partial t}\left(\begin{array}{c}
u(\zeta, t) \\
v(\zeta, t)
\end{array}\right) \operatorname{grad} \log \|z\|\right\} \\
=r D(u, v ; \xi, \eta)\left\{D(\xi, \eta ; x, y)+\left(\begin{array}{c}
\operatorname{Re} h(\zeta, t) \\
\operatorname{Im} h(\zeta, t)
\end{array}\right) \operatorname{grad} \log \|z\|\right\},
\end{gathered}
$$

where we have used (3.20), and the Cauchy-Riemann equations which are valid since $\|\zeta\|<1$. Further calculation leads to the formula

$$
\begin{gathered}
D(U, V ; x, y)= \\
=\|z\|^{-1} D(u, v ; \xi, \eta)\left\{I+r^{2}\left(\begin{array}{l}
\operatorname{Re}(h(\zeta, t)-\zeta) \\
\operatorname{Im}(h(\zeta, t)-\zeta)
\end{array}\right)(\xi, \eta)\right\},
\end{gathered}
$$


where $I$ denotes the $2 n \times 2 n$ identity matrix.

It is easy to verify that the matrix

$$
A=r^{2}\left(\begin{array}{l}
\operatorname{Re}(h(\zeta, t)-\zeta) \\
\operatorname{Im}(h(\zeta, t)-\zeta)
\end{array}\right)(\xi, \eta)
$$

has proportional columns, and hence $\operatorname{rank} A=1$. This implies that $\operatorname{det}(I+A)=1+\operatorname{trace} A$, and (3.10) yields that

$$
\begin{aligned}
\operatorname{det}(I+A)=1 & +r^{2} \operatorname{Re}\langle h(\zeta, t)-\zeta, \zeta\rangle=r^{2} \operatorname{Re}\langle h(\zeta, t), \quad \zeta\rangle \\
& \geq r^{2}\|\zeta\|^{2}\left(\frac{1-c\|\zeta\|}{1+c\|\zeta\|}\right) \geq \frac{1-c}{1+c} .
\end{aligned}
$$

Applying this to $(3.25)$ we obtain

$$
\begin{gathered}
|J(U, V ; x, y)| \geq\|z\|^{-2 n}\left(\frac{1-c}{1+c}\right)|J(u, v ; \xi, \eta)| \geqq \\
\|z\|^{-2 n}\left(\frac{1-c}{1+c}\right)|\operatorname{det} D f(\zeta, t)|^{2} .
\end{gathered}
$$

The last equality holds because $\zeta \in B^{n}$ and $f(\zeta, t)$ is holomorphic in $B^{n}$.

For the subordination chain (3.3) we have

$$
D f(\zeta, t)=e^{t} D f\left(\zeta e^{-t}\right)(I-E(\zeta, t)), \quad t=\log \|z\|,
$$

and this with the quasiconformality, (2.1), of $f$ yields

$$
K|\operatorname{det} D f(\zeta, t)| \geq\|z\|^{n}\left\|D f\left(\zeta e^{-t}\right)\right\|^{n}|\operatorname{det}(I-E(\zeta, t))| \text {. }
$$

The inequalities $1-c \leq\|I-E(\zeta, t)\| \leq 1+c$ and familiar results about the distortion of linear operators lead to

$$
\|I-E(\zeta, t)\|^{2 n} /|\operatorname{det}(I-E(\zeta, t))|^{2} \leq\left(\frac{1+c}{1-c}\right)^{2 n-2} .
$$

From (3.26) - (3.29) we obtain the inequality

$$
|J(U, V ; x, y)| \geq K^{-2}\left(\frac{1-c}{1+c}\right)^{2 n-1} \frac{\|D f(\zeta, t)\|^{2 n}}{\|z\|^{2 n}} .
$$

We return to $(3.25)$ and observe that

$$
\begin{gathered}
\|D(U, V ; x, y)\| \leq\|z\|^{-1}\|D(u, v ; \xi, \eta)\|\|I+A\| \\
=\|z\|^{-1}\|D f(\zeta, t)\|\|I+A\| .
\end{gathered}
$$

The equality $\|D(u, v ; \xi, \eta)\|=\|D f(\zeta, t)\|$ between the operator norm in $\mathcal{L}_{\mathcal{L}}\left(R^{2 n}\right)$ of the $2 n \times 2 n$ real matrix on the left and the $\mathscr{L}\left(C^{n}\right)$ norm of the $n \times n$ complex matrix on the right follows from the holomorphy since 
$\zeta \in B^{n}$. (This is an easy calculation involving the Cauchy-Riemann equations and the two operators with their respective norms.)

Next we derive an upper bound for the $\mathscr{L}\left(R^{2 n}\right)$ norm $\|I+A\|$. Let $a=\left(a_{1}, \ldots, a_{2 n}\right) \in R^{2 n}$ and observe that

$$
A\left(\begin{array}{c}
a_{1} \\
\vdots \\
a_{2 n}
\end{array}\right)=r^{2}\left(\begin{array}{c}
\operatorname{Re}(h(\zeta, t)-\zeta) \\
\operatorname{Im}(h(\zeta, t)-\zeta)
\end{array}\right)\left\langle(\xi, \eta),\left(a_{1}, \ldots, a_{2 n}\right)\right\rangle
$$

With the Schwarz inequality we obtain

$$
\|(I+A) a\| \leq(1+r\|h(\zeta, t)-\zeta\|)\|a\|,
$$

where we have also used the fact that $\|\zeta\|=1 / r$. The form, (3.7), of $h(\zeta, t)$ enables us to write

$$
h(\zeta, t)-\zeta=2 E(\zeta, t)(I-E(\zeta, t))^{-1}(\zeta),
$$

from which we infer that

$$
\|h(\zeta, t)-\zeta\| \leq 2 c /((1-c) r) .
$$

It follows that

$$
\|I+A\| \leq(1+c) /(1-c) .
$$

Finally, (3.24) follows from $(3.30)-(3.32)$ and the proof of Theorem 3.1 is complete.

Remarks. A difficulty that arises when one attempts to check the condition (3.11),

$$
\left(1-\|z\|^{2}\right)\left\|(D f(z))^{-1} D^{2} f(z)(z, \cdot)\right\| \leq c,
$$

is the problem of estimating the norm of the inverse $(D f(z))^{-1}$. However, a result by Kato [5] shows that $\left\|(D f)^{-1}\right\| \leq\|D f\|^{n-1} /|\operatorname{det} D f|$. Hence if $f$ satisfies (2.1) and the condition

$$
\left(1-\|z\|^{2}\right)\left\|D^{2} f(z)(z, \cdot)\right\| /\|D f(z)\| \leq c / K,
$$

holds for some $c<1$ then (3.11) will follow.

Example. If we let $h\left(z_{1}\right)=z_{1}\left(e^{a z_{1}}-1\right)$ in the example (2.17) then the resulting map $f(z)$ is holomorphic and univalent in $C^{2}$. Clearly $f$ is quasiregular in $B^{n}$ since $h^{\prime}\left(z_{1}\right)$ is bounded in $\left|z_{1}\right|<1$. Furthermore for $|a|$ sufficiently small $f$ will satisfy (3.11) for some $c<1$ since

$$
\left\|(D f(z))^{-1} D^{2} f(z)(z, \cdot)\right\|=|a|\left|\left(2+a z_{1}\right) z_{1} e^{a z_{1}}\right| .
$$

Thus $f$ admits a quasiconformal homeomorphic extension to $R^{2 n}$, but the extension cannot agree with the original holomorphic $f$ throughout $C^{n}$. This follows from a result of A. Marden and S. Rickman (Proc. Amer. Math. Soc. 46, 1974, 226-228) that says a nonlinear holomorphic mapping of $C^{n}(n \geq 2)$ cannot be quasiregular in a neighborhood of infinity. 


\section{References}

[1] BeCker, J.: Löwnersche Differentialgleichung und quasikonform fortsetzbare schlichte Funktionen. - J. Reine Angew. Math. 255, 1972, 23-43.

[2] Gold berg, M. A.: The derivative of a determinant. - Amer. Math. Monthly 79, $1972,1124-1126$.

[3] Golusin, G. M.: Geometrische Funktionentheorie. - Deutscher Verlag der Wissen. schaften, Berlin, 1957.

[4] HaHN, K. T., and J. MrtcheLL: Representation of linear functionals on $H^{p}$ spaces over bounded symmetric domains in $C^{n} \quad(n>1)$ for $0<p<1$. . Proc. A.M.S. Summer Institute on Differential Geometry 1973.

[5] Kato, T.: Estimation of iterated matrices, with application to the von Neumann condition. - Numer. Math. 2, 1960, 22-29.

[6] LöwNER, K.: Untersuchungen über schlichte konforme Abbildungen des Einheits. kreises. - Math. Ann. 89, 1923, 103-121.

[7] Pfaltzgrafr, J. A.: Subordination chains and univalence of holomorphic maps in $C^{n}$. - Math. Ann. 210, 1974, 55-68.

[8] Pommerenke, Ch.: Über die Subordination analytischer Funktionen. - J. Reine Angew. Math. 218, 1965, 159-173.

[9] VärsäLÄ, J.: Lectures on n-dimensional quasiconformal mappings. - Lecture Notes in Mathematics 229, Springer-Verlag, Berlin - Heidelberg New York, 1971.

University of North Carolina-Chapel Hill

Department of Mathematics

Chapel Hill

North Carolina 27514, USA

Received 18 March 1974 